\title{
Strategies for prompt searches for GRB afterglows: The discovery of the GRB 001011 optical/near-infrared counterpart using colour-colour selection ${ }^{\star}$
}

J. Gorosabel ${ }^{1,2}$, J. U. Fynbo ${ }^{2}$, J. Hjorth ${ }^{3,4}$, C. Wolf ${ }^{5}$, M. I. Andersen ${ }^{6}$, H. Pedersen ${ }^{3}$, L. Christensen $^{3}$, B. L. Jensen ${ }^{3}$, P. Møller ${ }^{2}$, J. Afonso ${ }^{7}$, M. A. Treyer ${ }^{8}$, G. Mallén-Ornelas ${ }^{9}$, A. J. Castro-Tirado ${ }^{10,11}$,

A. Fruchter ${ }^{12}$, J. Greiner ${ }^{13}$, E. Pian ${ }^{14}$, P. M. Vreeswijk ${ }^{15}$, F. Frontera ${ }^{16}$, L. Kaper ${ }^{15}$, S. Klose $^{17}$,

C. Kouveliotou ${ }^{18}$, N. Masetti ${ }^{16}$, E. Palazzi ${ }^{16}$, E. Rol ${ }^{15}$, I. Salamanca ${ }^{15}$, N. Tanvir ${ }^{19}$,

$$
\text { R. A. M. J. Wijers }{ }^{20} \text {, and E. van den Heuvel }{ }^{15}
$$

1 Danish Space Research Institute, Juliane Maries Vej 30, 2100 Copenhagen $\varnothing$, Denmark e-mail: jgu@dsri.dk

2 European Southern Observatory, Karl-Schwarzschild-Straße 2, 85748 Garching, Germany e-mail: jfynbo@eso.org; pmoller@eso.org

3 Astronomical Observatory, University of Copenhagen, Juliane Maries Vej 30, 2100 Copenhagen $\varnothing$, Denmark e-mail: jens@astro.ku.dk; holger@astro.ku.dk; lise@astro.ku.dk; brian_j@astro.ku.dk

4 Observatoire Midi-Pyrénées (LAS), 14 avenue E. Belin, 31400 Toulouse, France

5 Max-Planck-Institut für Astronomie, Königstuhl 17, 69117 Heidelberg, Germany e-mail: cwolf@mpia-hd.mpg.de

${ }^{6}$ Division of Astronomy, PO Box 3000, 90014 University of Oulu, Finland e-mail: michael.andersen@oulu.fi

7 Blackett Laboratory, Imperial College, Prince Consort Road, London SW7 2BW, UK e-mail: j.afonso@ic.ac.uk

8 Laboratoire d'Astronomie Spatiale, Traverse du Siphon, BP 8, 13376 Marseille, France e-mail: Marie.Treyer@astrsp-mrs.fr

9 Department of Astronomy, University of Toronto, 60 St. George Street, Toronto, ON, M5S 3H8, Canada e-mail: mallen@astro.utoronto.ca

10 Laboratorio de Astrofísica Espacial y Física Fundamental (LAEFF-INTA), PO Box 50727, 28080 Madrid, Spain e-mail: ajct@laeff.esa.es

11 Instituto de Astrofísica de Andalucía (IAA-CSIC), PO Box 03004, 18080 Granada, Spain e-mail: ajct@iaa.es

12 Space Telescope Science Institute, 3700 San Martin Drive, Baltimore, MD 21218, USA e-mail: fruchter@stsci.edu

13 Astrophysikalisches Institut, Potsdam, Germany e-mail: jgreiner@aip.de

14 Osservatorio Astronomico di Trieste, via Tiepolo 11, 34131 Trieste, Italy e-mail: pian@tesre.bo.cnr.it

15 University of Amsterdam, Kruislaan 403, 1098 SJ Amsterdam, The Netherlands e-mail: pmv@astro.uva.nl; lexk@astro.uva.nl; evert@astro.uva.nl; isabel@astro.uva.nl; edvdh@astro.uva.nl

16 Istituto Tecnologie e Studio Radiazioni Extraterrestri, CNR, via Gobetti 101, 40129 Bologna, Italy e-mail: filippo@tesre.bo.cnr.it; masetti@tesre.bo.cnr.it; eliana@tesre.bo.cnr.it

17 Thüringer Landessternwarte Tautenburg, 07778 Tautenburg, Germany e-mail: klose@tls-tautenburg.de

18 NASA MSFC, SD-50, Huntsville, AL 35812, USA

e-mail: kouveliotou@eagles.msfc.nasa.gov

19 Department of Physical Sciences, University of Hertfordshire, College Lane, Hatfield, Herts AL10 9AB, UK e-mail: nrt@star.herts.ac.uk

${ }^{20}$ Department of Physics and Astronomy, State University of New York, Stony Brook, NY 11794-3800, USA e-mail: rwijers@astro.sunysb.edu

Received 19 September 2001 / Accepted 8 November 2001

Send offprint requests to: J. Gorosabel, e-mail: jgu@dsri.dk

* Based on observations collected at the European Southern Observatory, La Silla and Paranal, Chile (ESO Programmes 165.H-0464(A), 165.H-0464(E) and 165.H-0464(G)). 
Abstract. We report the discovery of the optical and near-infrared counterparts to GRB 001011. The GRB 001011 error box determined by Beppo-SAX was simultaneously imaged in the near-infrared by the 3.58-m New Technology Telescope and in the optical by the 1.54-m Danish Telescope $\sim 8 \mathrm{hr}$ after the gamma-ray event. Here we implement the colour-colour discrimination technique proposed by Rhoads (2001) and extend it using near-IR data as well. We present the results provided by an automatic colour-colour discrimination pipe-line developed to discern the different populations of objects present in the GRB 001011 error box. Our software revealed three candidates based on single-epoch images. Second-epoch observations carried out $\sim 3.2$ days after the burst revealed that the most likely candidate had faded, thus identifying it with the counterpart to the GRB. In deep $R$-band images obtained 7 months after the burst a faint $(R=25.38 \pm 0.25)$ elongated object, presumably the host galaxy of GRB 001011, was detected at the position of the afterglow. The GRB 001011 afterglow is the first discovered with the assistance of colour-colour diagram techniques. We discuss the advantages of using this method and its application to error boxes determined by future missions.

Key words. galaxies: fundamental parameters - galaxies: statistics - gamma rays: bursts - techniques: photometric quasars: general

\section{Introduction}

Gamma-ray Bursts (GRBs) are intense flashes of high energy photons that occur uniformly distributed on the sky. They were discovered in 1967 (see Bonnell \& Klebesadel 1996 for a discussion of the first GRB detections), but due to the lack of rapid and precise localisations, their emission at other wavelengths was not detected until 1997 (van Paradijs et al. 1997; Frail et al. 1997; Costa et al. 1997). Thus, for 30 years they were not localised at longer wavelengths (X-ray, UV, optical, IR, radio) and their distances could not be measured. The breakthrough that occurred in 1997 can be attributed to the advent of the Beppo-SAX X-ray satellite (Boella et al. 1997), thanks to its fast (within a few hours) and precise (few arcmin error radius) localisations. Since the determination of the redshift of GRB 970508 (Metzger et al. 1997) another 17 secure spectroscopic redshifts have been determined to date (not considering the supernova SN1998bw; Galama et al. 1998), ranging from $z=0.43$ (Vreeswijk et al. 2001; Hjorth et al. 2000a, 2000b) to $z=4.50$ (Andersen et al. 2000). For an additional 8 GRBs, optical counterparts have been found, but with no conclusive published redshift determinations.

It is now widely accepted that at least the longduration GRBs originate at cosmological distances (with the exception of GRB 980425) with isotropic equivalent energy releases ranging from $10^{51}$ to $10^{54} \mathrm{erg}$ (see van Paradijs et al. 2000 for a review). Frail et al. (2001) have recently claimed that considering corrections for possible beaming effects the range of the inferred high-energy release is restricted to a narrower band around $10^{51} \mathrm{erg}$. Current models invoke the collapse of a massive star into a black hole (Woosley 1993; Paczyński 1998) or the merging of two compact objects (e.g. Lattimer \& Schramm 1974). The intrinsic brightness of GRBs and their afterglows allows one to probe the nature of their distant host galaxies and potentially the star-formation history of the early universe (Lamb \& Reichart 2000).

Since the first detection of an optical counterpart to a GRB, most searches have been based either on comparing a single epoch image to Digital Sky Survey (DSS) images in order to search for new objects or by comparing images taken through the same filter at different epochs in order to find fading, transient objects. However, many optical transients are fainter than the DSS limit at the time of the first optical follow-up observations, and in some cases the afterglow light curves show "plateaus" that could disguise their transient nature (GRB 000301C, Masetti et al. 2000, GRB 001007, Castro Cerón et al. 2001) or even brightness increases (GRB 970508; Castro-Tirado et al. 1998; Pedersen et al. 1998). Hence, it is clear that the identification process would benefit from alternative identification techniques. One such alternative is using colourcolour selection techniques similar to those used for quasar selection for many years (e.g. Warren et al. 1991 and references therein). Before the Beppo-SAX era colour-colour diagrams were applied to deep late-time CCD images with the purpose of detecting the presence of quiescent objects with anomalous colours within GRB error boxes. When the probability of finding one of these objects in a small GRB error box was low $\left(<10^{-3}\right)$ the object was considered potentially GRB related. Examples of such objects are extragalactic (e.g. quasars and AGNs; Vrba et al. 1995; Luginbuhl et al. 1995) as well as Galactic (e.g. neutron stars; Sokolov et al. 1995, flare stars; Gorosabel \& Castro-Tirado 1998, white dwarfs; Motch et al. 1990, novae; Zharykov et al. 1995).

The first reported attempt to exploit colour-colour diagrams for early afterglow identification of GRBs was carried out at the beginning of the Beppo-SAX era with optical images of GRB 970111 taken 19 hours after the trigger (Gorosabel et al. 1998a). However, GRB 970111 did not show any detectable optical emission and the feasibility of colour-colour discrimination techniques was not demonstrated. Recently, Rhoads (2001) has presented a detailed discussion of colour-colour discrimination techniques and the feasibility of using them to distinguish GRB power-law spectral energy distributions from curved (black-body) stellar spectra. In an a posteriori analysis, Śimon et al. (2001) show afterglow optical colours cluster in a precise position on the optical colour-colour diagrams. In this paper we present the result of implementing such principles by applying an automatic colour-colour discrimination software pipe-line to data taken for GRB 001011 just $\sim 8$ hours after the trigger. We demonstrate that the colour selection techniques can be successfully applied to identify GRB afterglows, including the use of near-IR data. 
In Sect. 2 we describe the observations of the GRB 001011 error-circle. In Sect. 3 we detail a candidateselection method based on the study of the optical/nearinfrared colours of the objects in the field. We illustrate the technique by describing its application to GRB 001011, which enabled the discovery of its optical/near-infrared counterpart. Section 4 shows the characteristics of the GRB 001011 afterglow and its likely host galaxy. Finally, in Sects. 5 and 6 we discuss and list the conclusions of our work.

\section{Observations}

GRB 001011 was detected on October 11.6631 UT 2000 by both the Wide Field Cameras (WFC) and the GammaRay Burst Monitor (GRBM) on board the Beppo-SAX satellite and localised with an accuracy of $5^{\prime}$ (Gandolfi et al. 2000a). The position was later refined to $2^{\prime}$ prior to our observations $\left(\alpha_{2000}=18^{\mathrm{h}} 23^{\mathrm{m}} 4.32^{\mathrm{s}}, \quad \delta_{2000}=\right.$ $-50^{\circ} 53^{\prime} 56^{\prime \prime}$. 4 ; Gandolfi et al. 2000b).

Optical ( $R$-band) and near-infrared ( $J$ and $K$ s bands) observations were carried out with the 1.54-m Danish Telescope (1.54D) and the 3.58-m New Technology Telescope (NTT), both at ESO, La Silla, on October 11.9700-12.0361 UT, between 7.37 and 8.95 hours after the burst. Optical and near-infrared comparison images were obtained with the same telescopes during the following week. Deep $R$-band images were obtained at the $1.54 \mathrm{D}$ in April 2001 and at the 8.2-m UT1 of ESO's Very Large Telescope (VLT) in May 2001, 6-7 months after the burst. Table 1 displays the observing log.

The near-infrared NTT observations were made with the infrared spectrograph and imaging camera "Son OF Isaac" (SOFI) using both the Large Field mode (LF, October 11) and the Small Field mode (SF, October 17). The fields of view (FOVs) in SF and LF modes are $2.4 \times 2.4$ and $4.9 \times 4.9$, respectively. The $1.54 \mathrm{D}$ observations were made with the Danish Faint Object Spectrograph and Camera (DFOSC) which provides a FOV of $13 ! 7 \times 13 ! 7$. Consequently, both the optical and near-infrared images taken a few hours after the GRB trigger covered the entire refined WFC error box. The last set of deep images were acquired with the FOcal Reducer and Spectrograph (FORS1) mounted at UT1 (Antu) of the VLT.

\subsection{Photometric calibration}

The field was calibrated in the near-infrared using observations of the standard stars sj9178, sj9013, and sj9106 at different airmasses. These standards and thus the nearIR measurements reported in the present paper are based on the JHKs photometric system introduced by Persson et al. (1998). The derived zero-point error was 0.03 mag in both the $J$ and $K \mathrm{~s}$ bands. In the optical the field was calibrated using observations of the Landolt field SA 107 at different airmasses (Landolt 1992). The zero-point error for the $R$-band was estimated to be 0.02 mag. In Table 2 we give the $R J K \mathrm{~s}$ magnitudes for 6 secondary standard stars in the GRB 001011 field (see Fig. 1). The photometry of these stars was performed using the DAOPHOT-II package (Stetson 1987, 1997). The $R J K$ s zero-point errors were added in quadrature to the measurement error derived by the DAOPHOT-II package, giving the error in the magnitudes shown in Tables 1 and 2. Stars \#1, \#5, and \#6 correspond to the USNO-A2.0 stars U0375-35233918, U0375-35222053, and U0375-35221378, which are listed to have $R$-band magnitudes of $15.3,16.1$, and 16.3 , respectively. Using our calibration we find $R=15.64 \pm 0.04$, $R=16.24 \pm 0.04$ and $R=16.33 \pm 0.04$ (see Table 2). Based on these three stars we have calculated a mean $R$ band offset of $0.17 \mathrm{mag}$ with respect to the USNO-A2.0 catalogue. Therefore, the calibration used in the present paper is offset by $0.17 \mathrm{mag}$ relative to the preliminary mean zero-point based on the USNO-A2.0 catalogue, which was used in the discovery report (Gorosabel et al. 2000).

\section{Candidate selection using colour-colour diagrams}

The main principle behind colour selection of optical afterglows (OAs) is that they have power-law spectral energy distributions, which can be distinguished from curved thermal stellar spectra in colour-colour plots. This is a principle that has been used to select quasars for many years (Warren et al. 1991 and references therein). This fact can be used to carry out a first colour-based selection of OA candidates. These candidates can subsequently be checked by second epoch observations aimed at detecting variability. The advantage of having an identification scheme that is not based on the transient nature of OAs is that it allows fast follow-up spectroscopy or polarimetry, also for OAs that are fainter than the DSS limit.

All afterglows observed so far as well as theoretical fireball (e.g. Sari et al. 1998; Mészáros 2001; Piran 2001) or cannonball models (Dado et al. 2001) show that GRB optical/infrared afterglow decays are reasonably well described by a spectral index independent of time $\left(F_{\nu} \sim\right.$ $\nu^{-\beta}, \beta$ not a function of time). Thus, the colours should remain approximately constant with time, and the technique is applicable any time after the GRB. In the case that $\beta$ is a function of time, then the afterglow decay is not achromatic. Thus, if the images used to derive the colours are very separated in time and $\beta$ varies strongly with time (extremely unlikely for GRB afterglows), the magnitudes can not be shifted easily to the same epoch assuming a power law decay $F_{\nu} \sim t^{-\alpha}$, with an unique achromatic value of $\alpha$ for all the bands. However, if the images are contemporaneous (or at least quasi-simultaneous as in the case of GRB 001011) the error introduced by the decay epoch-scaling factor is negligible, even if $\beta$ depends on time (see Sect. 3.1 for colour error estimates due to the epoch-scaling factor). So, the technique is not restricted to achromatic afterglow decays, if quasi-simultaneous data are used. Therefore, the method should be valid at any epoch after the gamma-ray burst at least until the 


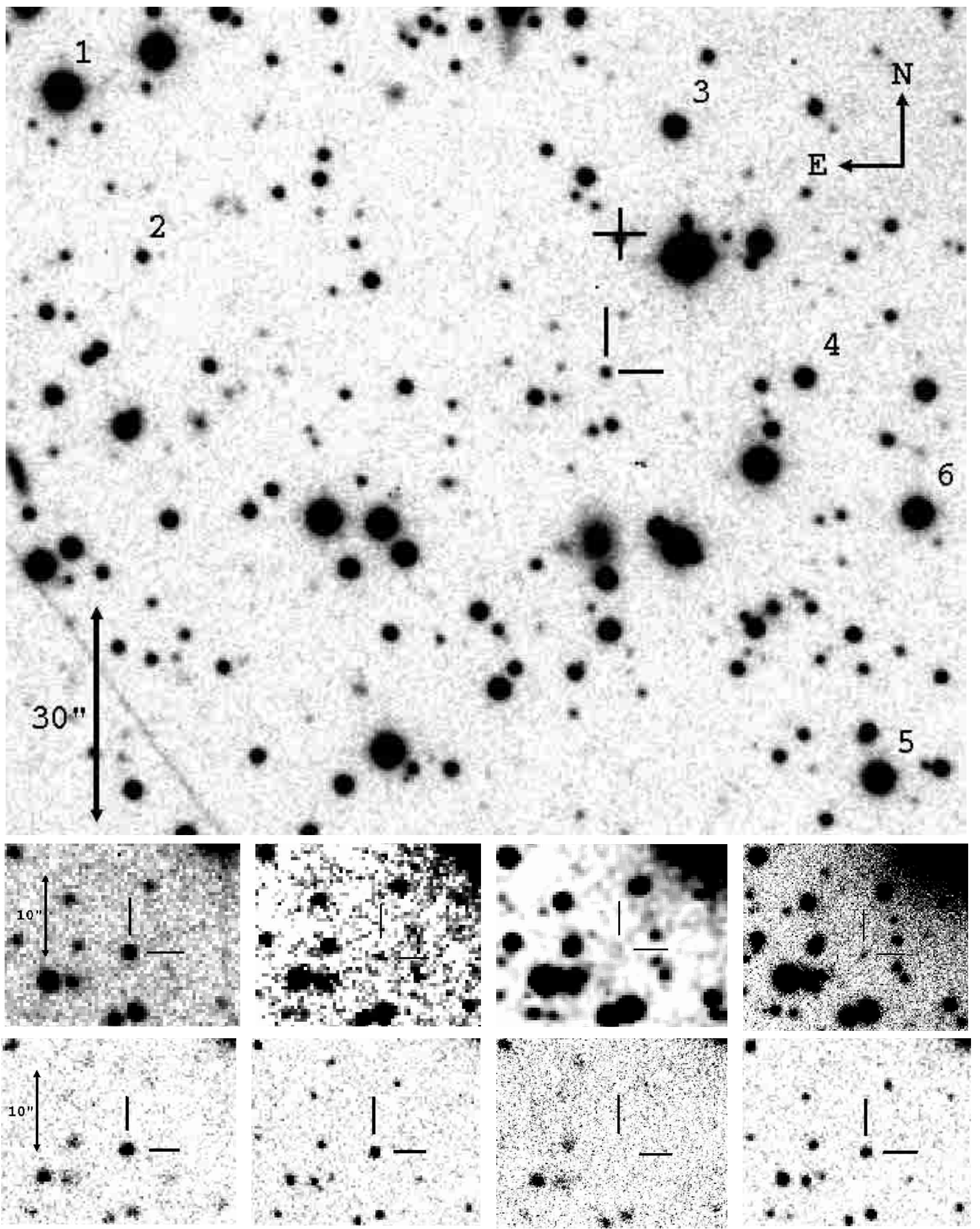

Fig. 1. Upper figure: $R$-band image of the counterpart (between tick-marks) and the secondary standards in the field (see Table 2). The cross indicates the position of the refined WFC error circle centre. Central row of figures: from the left to the right side the $R$-band decay is displayed; 11.9934-12.0361 UT Oct. 2000, 14.0029-14.0521 UT Oct. 2000, 19.3710-22.4149 UT April 2001 and 20.1618-20.1905 UT May 2001 (VLT detection of the host galaxy). Lower row of figures: the first three panels show the $K$ s-band brightness evolution; 11.9700-11.9828 UT, 11.9964-12.0092 UT and 17.9794-18.0138 UT Oct. 2000. The fourth figure displays the $J$-band detection on 11.9834-11.9959 UT Oct. 2000. 

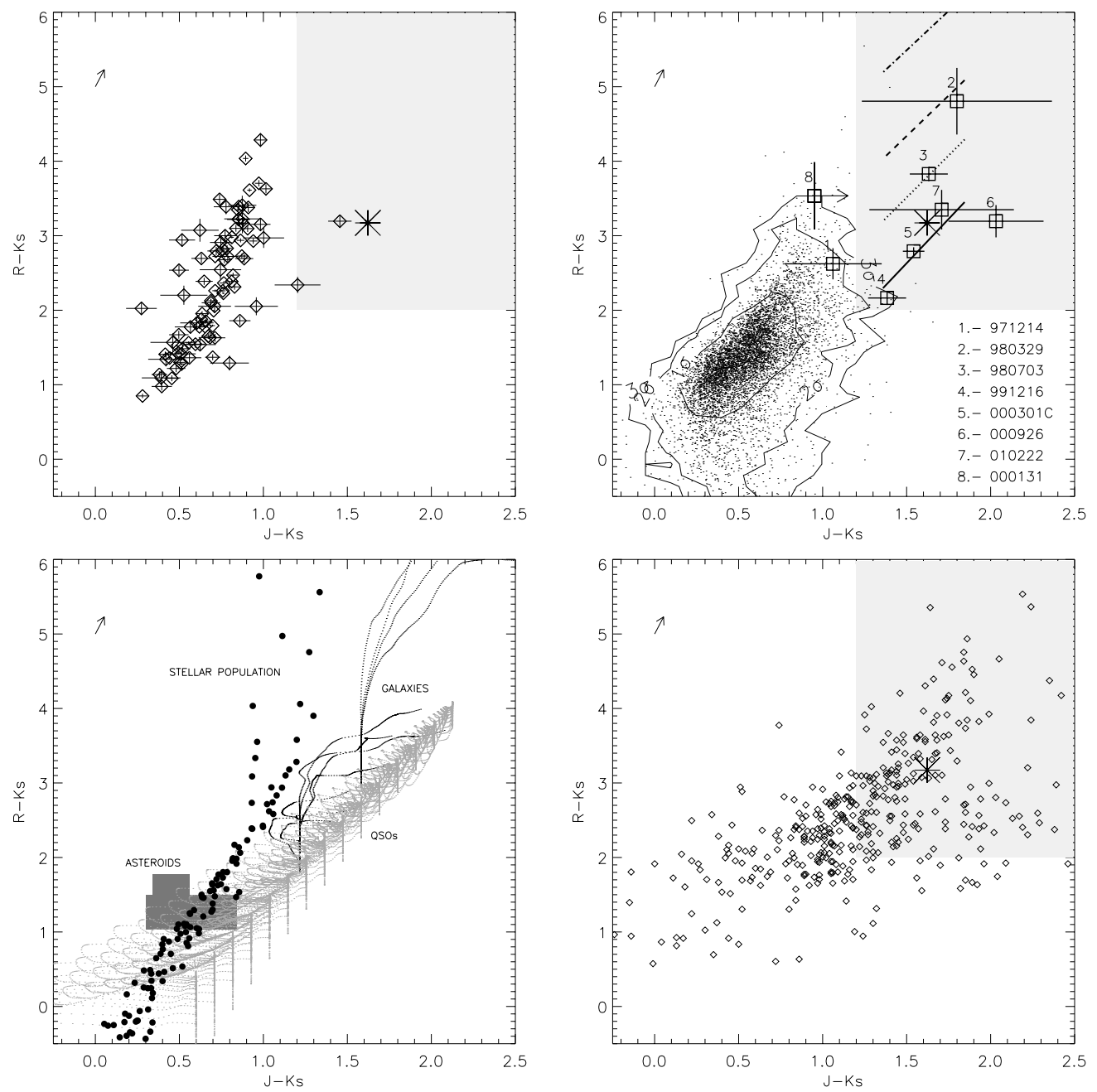

Fig. 2. Upper left panel: the panel shows an $R-K$ s versus $J-K$ s colour-colour diagram for the objects inside the GRB 001011 error box (open diamonds). The star represents the colours of the GRB 001011 optical/near-IR counterpart. The errors of the counterpart colours are smaller than the size of the star. The shaded region shows the OAs colour-colour space locus determined by our selection criteria $R-K \mathrm{~s}>2.0, J-K \mathrm{~s}>1.2$. As can be seen, the colours of the GRB 001011 counterpart are consistent with the shaded area and inconsistent with almost all the other objects within the error circle. Only two non-transient objects in the error box have colours consistent with the shaded area. They are very likely quasars or compact galaxies (see Sect. 3.4). Upper right panel: the figure shows an $R-K \mathrm{~s}$ vs. $J-K \mathrm{~s}$ colour-colour diagram for the $100972 \mathrm{MASS}+\mathrm{USNO}$ sources (dots) found in a $\pm 5^{\circ}$ window around GRB 001011. As it is shown, the counterpart (star) exhibits colours inconsistent at least at a $3 \sigma$ level with the objects in the 2MASS+USNO catalogue (see Sect. 3.1 for information on the iso-density contour levels). As can be seen, the 2MASS+USNO catalogue traces most of the colours shown in the upper left panel. The seven open squares labelled from 1 to 7 represent the colours of afterglows with measured $R-K \mathrm{~s}$ and $J-K \mathrm{~s}$ colours to date. The additional square labelled with number 8 represents GRB 000131 which was not detected in the $J$-band (Andersen et al. 2000). As can be seen, at least seven of the eight open squares and the star are consistent with the shaded area. The eighth open square (GRB 000131) is likely consistent with this region too. The solid straight line inside the shaded region represents the colour trace of a pure power-law SED with $\beta$ ranging from 0.6 to 1.5. When Lyman- $\alpha$ blanketing is taken into account the pure power-law SED (straight solid line) is shifted upwards (three dashed lines). From the bottom to the top, the three dashed lines show the colours of a power-law SED when the Lyman- $\alpha$ blanketing is considered for an afterglow at $z=5.0,5.5$ and 6.0, respectively. As can be seen, the higher the redshift the more distant the power-law SED from the stellar trace is. Lower left panel: the figure displays synthetic colour-colour traces for stars (filled circles), galaxies (dashed curves) and quasars (dotted curves). The shaded polygonal background region represents the loci occupied by the asteroid samples of McCheyne et al. (1985) and Gaffey et al. (1993). As can be seen, the bunch of objects in the left upper panel (located outside the shaded area) can be reproduced by asteroid, stellar, galaxy and quasar synthetic colours. However, the colours inside the shaded area can only be obtained by galaxy and quasar templates, which makes these objects a potential source of contamination. Most of the galaxies can however be eliminated because of their non-stellar profile. Lower right panel: we have over-plotted the colours of the galaxies from the NTT deep field (Fontana et al. 2000) to illustrate the problem of the contamination by galaxies (see Sect. 3.4). About $45 \%$ of the galaxies show colours consistent with the ones of OAs (shaded rectangle). General: in the four panels the length of the arrow and its orientation show the magnitude of Galactic extinction $\left(A_{R-K \mathrm{~s}}=0.23, A_{J-K \mathrm{~s}}=0.05\right)$ and its direction in the colour-colour space, respectively. All sources have been dereddened with the corresponding Galactic extinction given by Schlegel et al. (1998). 
Table 1. Journal of observations of the GRB 001011 optical/near-IR counterpart.

\begin{tabular}{|c|c|c|c|c|c|}
\hline Telescope & Date UT & $\begin{array}{c}\text { Seeing } \\
(\operatorname{arcsec})\end{array}$ & Filter & $\begin{array}{c}\text { Exp. Time } \\
\text { (s) }\end{array}$ & Mag. \\
\hline$\overline{\mathrm{NTT}}(+\mathrm{SOFI}, \mathrm{LF})$ & $11.9700-11.9828 / 10 / 2000$ & 1.5 & $K \mathrm{~s}$ & $15 \times 60$ & $17.56 \pm 0.05$ \\
\hline $\mathrm{NTT}(+\mathrm{SOFI}, \mathrm{LF})$ & $11.9834-11.9959 / 10 / 2000$ & 0.9 & $J$ & $15 \times 60$ & $19.26 \pm 0.05^{\dagger}$ \\
\hline NTT(+SOFI, LF $)$ & $11.9964-12.0092 / 10 / 2000$ & 0.7 & $K \mathrm{~s}$ & $15 \times 60$ & $17.58 \pm 0.04^{\dagger}$ \\
\hline $1.54 \mathrm{D}(+\mathrm{DFOSC})$ & $11.9934-12.0361 / 10 / 2000$ & 1.2 & $R$ & $5 \times 600$ & $20.99 \pm 0.05^{\dagger}$ \\
\hline $1.54 \mathrm{D}(+\mathrm{DFOSC})$ & $14.0029-14.0521 / 10 / 2000$ & 1.0 & $R$ & $5 \times 600$ & $23.74 \pm 0.23$ \\
\hline NTT $(+$ SOFI, SF $)$ & $17.9794-18.0138 / 10 / 2000$ & 1.0 & $K \mathrm{~s}$ & $30 \times 60$ & $>21.3^{\star}$ \\
\hline $1.54 \mathrm{D}(+\mathrm{DFOSC})$ & $19.3710-19.4064 / 04 / 2001$ & 0.9 & $R$ & $10 \times 200$ & $>24.3^{\star}$ \\
\hline $1.54 \mathrm{D}(+\mathrm{DFOSC})$ & $20.3647-20.4242 / 04 / 2001$ & 0.8 & $R$ & $14 \times 200$ & $>24.6^{\star}$ \\
\hline $1.54 \mathrm{D}(+\mathrm{DFOSC})$ & $22.3741-22.4149 / 04 / 2001$ & 1.1 & $R$ & $6 \times 600$ & $>24.5^{\star}$ \\
\hline $\mathrm{VLT}(+\mathrm{FORS} 1)$ & $20.1618-20.1905 / 05 / 2001$ & 0.9 & $R$ & $8 \times 300$ & $25.38 \pm 0.25$ \\
\hline
\end{tabular}

Table 2. Secondary standards in the field of GRB 001011.

\begin{tabular}{lllccc}
\hline & RA(J2000) & Dec(J2000) & $K \mathrm{~s}$ & $J$ & $R$ \\
\hline 1 & $18: 23: 12.45$ & $-50: 53: 34.4$ & $13.91 \pm 0.03$ & $14.33 \pm 0.03$ & $15.64 \pm 0.04$ \\
2 & $18: 23: 11.34$ & $-50: 53: 57.7$ & $16.47 \pm 0.06$ & $17.34 \pm 0.05$ & $20.26 \pm 0.06$ \\
3 & $18: 23: 03.47$ & $-50: 53: 41.8$ & $15.46 \pm 0.04$ & $16.17 \pm 0.04$ & $17.59 \pm 0.04$ \\
4 & $18: 23: 01.65$ & $-50: 54: 17.0$ & $15.92 \pm 0.04$ & $16.71 \pm 0.06$ & $18.38 \pm 0.05$ \\
5 & $18: 23: 00.72$ & $-50: 55: 13.3$ & $14.90 \pm 0.03$ & $15.28 \pm 0.03$ & $16.24 \pm 0.04$ \\
6 & $18: 23: 00.04$ & $-50: 54: 36.4$ & $14.97 \pm 0.03$ & $15.36 \pm 0.03$ & $16.33 \pm 0.04$ \\
\hline
\end{tabular}

emission of the host galaxy becomes dominant, usually weeks after the gamma-ray event.

\subsection{Description and implementation of an identification pipe-line}

Due to the transient nature of OAs it is of foremost importance to have a fast identification method once a GRB localisation has been released. It is therefore very important to try to find an optimal strategy for the identification of the OA. The most widely used strategy during the last three years has been to obtain an image, most often in the $R$-band, and then to look for new sources by comparing with DSS images (e.g., GRB 980425, GRB 980519, GRB 990123, GRB 990510, GRB 000301C, GRB 000926). In many cases this method may still be a fast and efficient way to localise the burst, but it is limited to the brightest OAs in fields of relatively little crowding.

Colour-colour selection is an ideal complement to the comparison with DSS images since it can be done with one-epoch observations and therefore allows fast follow-up spectroscopy or polarimetry. We have therefore designed a fast reduction pipe-line that constructs colour-colour diagrams of objects in GRB error boxes. Before the pipe-line can be used we need to obtain three nearly simultaneous images taken in the $R, J$ and $K$ s filters. This requires the use of more than one telescope and a possibility for fast data transfer, but for major modern observatories such as e.g. ESO and with modern network facilities this is not a serious limitation. The three images are then WCS (World Coordinate System; e.g. Mink 1997) calibrated and fed to the pipe-line. The error introduced in the colours $C$ by considering quasi-simultaneous images instead of simultaneous ones is of the order of $\Delta C \sim \alpha \frac{\Delta T}{\bar{T}}$, where $\Delta T$ is the total time-span covered by the set of images, $\bar{T}$ is the mean delay of the observation with respect to the gamma-ray event and $\alpha$ is the instant power-law decay slope $\left(F_{\nu} \sim t^{-\alpha}\right)$. In case the time-span covered in the observations $\Delta T$ is a non-negligible fraction of the delay $\bar{T}$, then all the measurements should be shifted to the same epoch assuming a value of $\alpha$. In the case of GRB 001011 the colour-colour diagram was constructed using $R, J$, and $K$ s-band images which overlap in time (indicated with a dagger in Table 1). For a value of $\alpha=1.33$ (as was found in the optical, see Sect. 4) the errors in the colours are $\Delta C \lesssim 0.1 \mathrm{mag}$.

The pipe-line uses several selection criteria or filters to improve the identification of candidates. The first of these filters is to reject sources outside the GRB error box. Once the spatially coincident sources have been found, aperture photometry is performed in the three images and a colourcolour diagram is constructed for all the objects inside the error box. The colours of the objects are dereddened using the Galactic extinction given by Schlegel et al. (1998) in the direction of the GRB error box centre. In addition, the pipe-line creates a list of high-redshift OA candidates, 
consisting of those sources detected in the two reddest bands but absent in the blue one.

For a quick analysis, the discrimination algorithm can be flagged to only use the relative colour differences among objects in the GRB error box. We can derive the twodimensional probability distribution of the uncalibrated colours in the field and construct the iso-density contour levels. Therefore, given an uncalibrated candidate we can determine the probability that this object belongs to the calculated probability distribution, i.e., whether its colours are typical of objects in the error box or not. This relative comparison largely removes the systematic effect of the Galactic reddening and also eliminates the influence of a variable (and colour dependent) transparency of the atmosphere, encountered during adverse observing conditions. Therefore, the colour-colour discrimination technique does not strictly require absolute photometric calibration. The upper left panel of Fig. 2 shows an $R-K \mathrm{~s}$ versus $J-K$ s colour-colour diagram for the objects inside the GRB 001011 error box (open diamonds). As can be seen, the colours of the GRB 001011 counterpart (star) are inconsistent with almost all the other objects within the error circle.

A more careful analysis requires a rough photometric calibration. A good representation of the distribution of colours of field stars is given by the 2MASS and USNOA2.0 catalogues (hereafter 2MASS+USNO), so the pipeline can be flagged to use this catalogue to obtain a well constrained sequence of non-transient point sources near the GRB position on the sky. In the top right panel of Fig. 2 we show with dots and contour levels the position of 10097 sources from the 2MASS+USNO database. These 2MASS+USNO sources were selected to be inside a $10^{\circ} \times 10^{\circ}$ window centred on the GRB 001011 error box coordinates. The size of the window has been chosen to contain $\sim 10^{4}$ sources, thus giving a large colour sample for the construction of the contour levels in a reasonably short computing time. In order to eliminate the possible reddening gradient inside the window each $2 \mathrm{MASS}+\mathrm{USNO}$ source has been dereddened by its individual reddening, rather than applying a mean reddening correction to all sources.

The 2MASS+USNO catalogue trace can be used to determine a preliminary colour calibration of the field. The colour calibration can simply be done by matching the median of the colours of the point-like sources in the field with the median of the 2MASS+USNO colours. By using the median rather than the average colour of the field objects, any bias introduced by objects with anomalous colours, which may be present in the field, is minimised. Only point-like sources are used to determine the median of the colours, so the effect of the possible presence of extended objects (galaxy clusters or nebular structures in general) is also eliminated. As seen in the upper right panel of Fig. 2 the median of the colour for the 2MASS+USNO catalogue is located at $J-K \mathrm{~s}=0.54$ and $R-K \mathrm{~s}=1.33$, respectively. The standard deviation in $(J-K \mathrm{~s})$ and $(R-K \mathrm{~s})$ is small, $0.35 \mathrm{mag}$ and $0.75 \mathrm{mag}$ respectively. For a typical Beppo-SAX error box size (10-100 $\left.\operatorname{arcmin}^{2}\right)$, at least 25 objects would be detected in all bands. Therefore, a colour zero-point for the field can be established with an accuracy of $0.07 \mathrm{mag}$ in $(J-K \mathrm{~s})$ and $0.15 \mathrm{mag}$ in $(R-K \mathrm{~s})$. This accuracy is fully sufficient for applying absolute colour selection criteria.

Most of the 2MASS+USNO sources are located in a sequence stretching from $(J-K \mathrm{~s}, R-K \mathrm{~s})=(0.1,0.5)$ to $(1,3)$. As we will describe in Sect. 3.3 the colours of the majority of the $2 \mathrm{MASS}+\mathrm{USNO}$ sources can be reproduced by stellar spectral templates.

\subsection{The colours of OAs and the selection criterion}

In the upper right panel of Fig. 2 we have over-plotted the colours of GRB 001011 (marked with a star) and the colours of OAs (open squares labelled from 1 to 7 ) with reported quasi-simultaneous $R, J$, and $K$ s-band magnitudes, i.e. GRB 971214 (\#1: Diercks et al. 1998; Ramaprakash et al. 1998), GRB 980329 (\#2: Fruchter 1999; Palazzi et al. 1998), GRB 980703 (\#3: Vreeswijk et al. 1999), GRB 991216 (\#4: Garnavich et al. 2000), GRB 000301C (\#5: Jensen et al. 2001), GRB 000926 (\#6: Fynbo et al. 2001a) and GRB 010222 (\#7: Masetti et al. 2001). Each GRB colour has been corrected for its Galactic foreground extinction (Schlegel et al. 1998). GRB 000131 was detected in the $R$ and $K_{\mathrm{s}}$-band but in the $J$-band only an upper limit was reported (Andersen et al. 2000). In order to visualise that the method is valid at least up to $z=4.5$ we have over-plotted with an arrow on an open square (labelled with an 8) the colour constraints of GRB 000131. As can be seen in Fig. 2 the GRB 000131 colours are likely consistent with the colours of the other seven OAs (shaded area). Most of these OAs have $R-K$ s and $J-K$ s colours that place them well away from the locus of the stellar sequence. For a given $R-K \mathrm{~s}$ colour the OAs are 0.5-1.0 mag redder in $J-K$ s than stars. The mean dereddened colours for the seven OAs are $\overline{(R-K \mathrm{~s})}_{\mathrm{GRB}}=3.3 \pm 0.9, \overline{(J-K \mathrm{~s})}_{\mathrm{GRB}}=1.6 \pm 0.3$. The positions of the known OAs in Fig. 2 do not show any correlation with their redshift.

The spectral energy distributions of GRB afterglows are fairly well described by pure power-laws in the optical to near-infrared range, $F_{\nu} \sim \nu^{-\beta}$. Using synthetic photometry we can therefore determine an analytic expression for the colour as function of $\beta$, which is independent of redshift:

$$
\begin{aligned}
& R-K \mathrm{~s}=1.525+\beta \times 1.284 \\
& J-K \mathrm{~s}=1.035+\beta \times 0.540
\end{aligned}
$$

In the right upper panel of Fig. 2 we have over-plotted a solid line that corresponds to a pure afterglow Spectral Energy Distribution (SED) with $\beta$ ranging from 0.6 to 1.5, as expected for afterglows. All the traces and objects (including afterglows) plotted in Fig. 2 have been dereddened by their corresponding Galactic extinction 


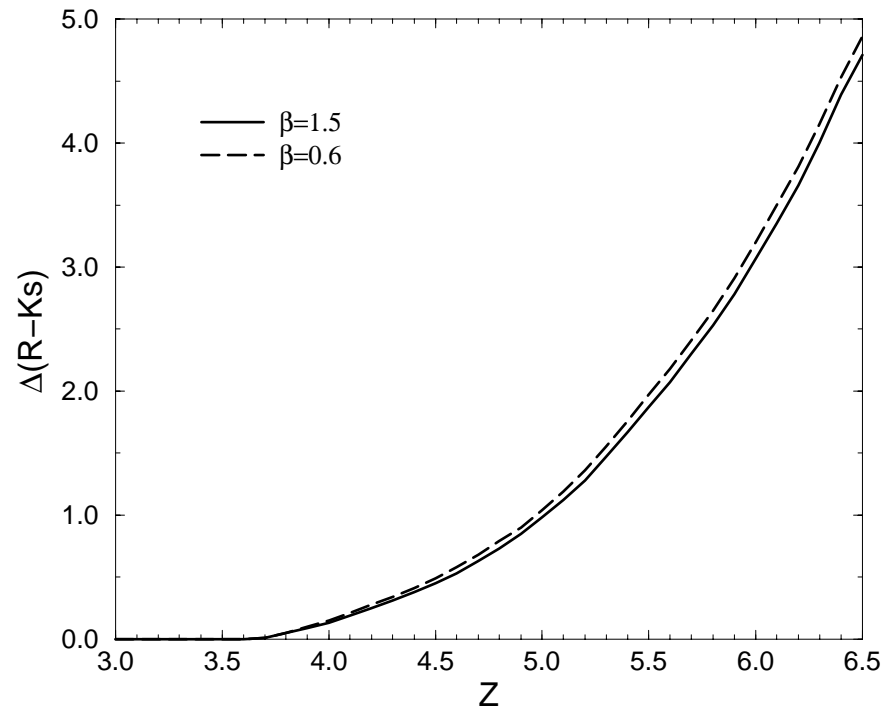

Fig. 3. The figure shows the reddening of the $R-K$ s colour of a pure power-law as a function of the redshift due to Lyman- $\alpha$ blanketing along the line of sight to the observer. The blanketing effects for the two extreme cases of $\beta=0.6$ (long-dashed line) and $\beta=1.5$ (solid line) are plotted. Most GRB afterglows are intermediate between these cases. The reddening of the $(R-K \mathrm{~s})$ colour due to the Lyman- $\alpha$ blanketing starts to be noticeable for redshifts $z>3.7$ (see text). For $z<3.7$ the blanketing effect is absent.

(Schlegel et al. 1998). As seen, the observed OAs are consistent with the predicted colours for power-law SEDs.

When $z \gtrsim 4$ the effect of Lyman- $\alpha$ blanketing becomes noticeable in the $R-K \mathrm{~s}$ colour, while the $J-K \mathrm{~s}$ colour is unaffected until $z \sim 8$. We have modelled the effect on the $R-K$ s colour by imposing Lyman- $\alpha$ blanketing on a power-law SED with spectral slopes in the range $0.6 \leq \beta \leq 1.5$ (Møller \& Jakobsen 1990; Madau 1995). The resulting spectrum was integrated for a range of redshifts, using the transmission function of an $R$-band filter as a weight function. The result is shown in Fig. 3. The effect of Lyman- $\alpha$ blanketing in the $R$-band has its onset at $z=3.7$ and is essentially independent of the intrinsic spectral slope. At a redshift of $z \sim 6.5$, the attenuation reaches $5 \mathrm{mag}$, implying that GRB afterglows are unlikely to be detected in the $R$-band at redshifts much beyond 6 (see Lamb \& Reichart 2000 for a discussion on the detectability of very high redshift GRBs).

The effect of the Lyman- $\alpha$ blanketing on the colourcolour space can be visualised on the upper right panel of Fig. 2. As the Lyman- $\alpha$ forest shifts into the $R$-band sensitivity wavelength region the value of $R-K$ s increases moving the solid straight-line (pure power-law) upwards (dotted, dashed and dash-dotted line). For simplicity we have only over-plotted the loci of a pure power-law affected by blanketing when the afterglow is located at $z=5.0$ (dotted line), 5.5 (dashed line) and 6.0 (dash-dotted line). As can be seen in Fig. 2 the effect of the blanketing is to further separate the colours expected for power-law SEDs from the non-transient objects.
In order to establish a list of OA candidates we use the following colour selection criteria: $R-K \mathrm{~s}>2.0, J-K \mathrm{~s}>$ 1.2. These criteria define the rectangular shaded area of Fig. 2. This area contains the expected colours for powerlaw OAs as well as OA SEDs modified by Ly- $\alpha$ blanketing and at the same time has very little contamination from the stellar sequence.

\subsection{Location of other astrophysical objects in the diagram}

In order to improve the discrimination method, the different regions which the non-transient classes of objects occupy in the colour-colour diagram must be determined. To achieve this goal we either use synthetic photometry with spectral templates from the literature (stars, galaxies and quasars, following Wolf et al. 2001a; Wolf et al. 2001b) or use colour observations from the literature (asteroids).

For stars we calculate $R-K \mathrm{~s}$ and $J-K$ s colours based on the spectral atlas of Pickles (1998), which contains spectra of 131 stars with spectral types ranging from O5 to M8. It covers different luminosity classes, but concentrates on main sequence stars, and it also contains some spectra for high metalicities. The colours for these 131 stars are shown with filled circles in the lower left panel of Fig. 2.

The expected colours for a population of galaxies were calculated using the galaxy spectral templates by Kinney et al. (1996). This library consists of ten SEDs averaged from integrated spectra of local galaxies ranging in wavelength from $125 \mathrm{~nm}$ to $1000 \mathrm{~nm}$. The input spectra of quiescent galaxies were sorted by morphology beforehand into four templates and the starburst galaxies were sorted by colour into six groups yielding six more templates. Colours were calculated for all individual spectra redshifted from 0 to 2.0 in steps of 0.01 . The derived galaxy colours are displayed in the lower left panel of Fig. 2 with dashed lines.

The quasar library is designed as a three-component model: We add a power-law continuum with an emissionline contour based on the template spectrum by Francis et al. (1991). The intensity of the emission-line contour was varied only globally, i.e. with no intensity dispersion among the lines. The slope of the power-law continuum $F_{\nu} \sim \nu^{-\beta}$ was varied in 15 steps of $\Delta \beta=0.2$ ranging from $\beta=-0.8$ to $\beta=2.0$. The library was calculated for 151 redshifts ranging in steps of $\Delta z=0.02$ from $z=0$ to $z=3$.

The number of quasars with redshift $z>3$ having $R \lesssim 23.5$ (the limiting magnitude of the discovery DFOSC image) expected inside the refined WFC error box is low $\left(\sim 10^{-2}\right.$; based on the contamination estimates given in Sect. 3.4 and in the fraction of $z>3$ quasars present in the $10^{\text {th }}$ recompilation by Véron-Cetty \& Véron 2001), therefore synthetic quasar colours have not been constructed for redshifts $z>3$. The synthetic quasar colours are represented with a dotted line in the lower left panel of Fig. 2. 
For satellites looking for GRBs in or near the ecliptic plane, such as HETE-II, it is also important to consider the contamination due to asteroids, especially as these objects do not appear in the sky surveys that are usually used (e.g. DSS). Based on the asteroid colour recompilation by McCheyne et al. (1985) we derived a mean colour of $\overline{(R-K \mathrm{~s})}=1.49 \pm 0.28$ and $\overline{(J-K \mathrm{~s})}=0.45 \pm 0.11$ based on a sample of seven asteroids. An independent sample of albedos of nine asteroids reported by Gaffey et al. (1993) gives $\overline{(R-K \mathrm{~s})}=1.27 \pm 0.23 \overline{(J-K \mathrm{~s})}=0.57 \pm 0.27$, consistent with the first sample. Thus, the location of asteroids in the $(J-K \mathrm{~s})$ vs. $(R-K \mathrm{~s})$ colour-colour diagram is far from the region where we find GRB afterglows, ensuring that the colour-colour technique is effectively screening out asteroids. The loci of the above mentioned two asteroid samples is shown by the shaded background region of the lower left panel of Fig. 2.

\subsection{Rejection of non-transient sources with similar colours as OAs}

Although there are many stars in the field, the stellar contamination is low. As can be seen in the upper right panel of Fig. 2 the GRB colour-colour space region (shaded rectangular area) is almost completely beyond the $3 \sigma$ contour level of the $2 \mathrm{MASS}+\mathrm{USNO}$ catalogue.

The overlap with galaxy colours is more significant. According to Huang et al. (2001) we would expect $\sim 4 \times 10^{3}, 10^{4}$ and $2.4 \times 10^{4}$ galaxies per square degree with $R<21, R<22$ and $R<23$, respectively. However, in the $21 \lesssim R \lesssim 23$ mag range only $\sim 20 \%$ of the galaxies are compact under observing conditions of 1."0 (Calar Alto Deep Imaging Survey, Wolf et al. 2001b). Thus, we have designed a selection filter to permit identification and elimination of all extended objects from the colour-colour plot. To apply this filter, the Full Width Half Maximum (FWHM) distribution for all objects in the field is derived. Next, the mode of the distribution is calculated, and all the objects beyond $\pm 3 \sigma$ from the FWHM mode can be rejected. Based on the NTT deep field catalogue (Fontana et al. 2000) we estimate that $\sim 45 \%$ of compact galaxies have colours consistent with GRBs (see the lower right panel of Fig. 2). Taking into account these estimates we would expect $\sim 1,3$ and 8 compact galaxies brighter than $R=21, R=22$ and $R=23$, respectively, in the 2 arcmin radius WFC error box. Thus, we can eliminate most of the galaxy contamination especially for bright OAs. This demonstrates that, besides the colour, the magnitude of the source is an important parameter that together with the colour shows the unusual nature of a source. However, we do not wish to exclude faint candidates since one of the important quests for future searches is to extend the detection sensitivity to fainter magnitudes (Fynbo et al. 2001b).

Quasars are not so easy to eliminate because they are point-like sources and their SEDs can be described by power-laws similar to those of afterglows. Fortunately (in this context), quasars are rare. According to Boyle et al. (1991), Hartwick \& Schade (1990) and Wolf et al. (1999) the number of quasars per square degree with $R<21$, $R<22$ and $R<23$ is $\sim 80,200$ and 400, respectively. The number of quasars inside the GRB 001011 error box would then be $0.3,0.7$ and 1.4, depending on the depth of the image $(R<21, R<22$ and $R<23)$. As mentioned, GRB afterglows exhibit spectral indices $\beta$ ranging from 0.6 to 1.5. Quasars instead show values of $\beta$ between 0 and 1 . Hence, afterglows tend to be redder than quasars. Therefore, the quasar contamination is mainly due to the reddest quasars. Only one third of the quasars show indices $\beta>0.6$ (Francis et al. 1991). The pipe-line is able to exploit this property and has an option to reject objects with spectral indices smaller than 0.6. The spectral index of each object is calculated by least-square fitting a power-law SED to the $R, J$, and $K$ s-band magnitudes.

Afterglows which are reddened by Lyman- $\alpha$ blanketing are shifted into a region of the colour-colour diagram which is occupied by L-type brown dwarfs. Although the density of brown dwarfs is low, of the order of one per square degree (Kirkpatrick et al. 1999; Fan et al. 2000) down to a $K$ s-band limiting magnitude of 20 , this complicates the secure colour-colour identification of high redshift afterglows in large error boxes.

Therefore, we consider that for a first selection of candidates the impact of contamination by non-transient sources is not severe, at least for GRB error boxes smaller than $\sim 10 \operatorname{arcmin}^{2}$ and possibly up to $\sim 100 \operatorname{arcmin}^{2}$.

\section{Results}

\subsection{Detection of the optical and near-infrared afterglow of GRB 001011}

In the upper left panel of Fig. 2 we show with open diamonds the $R-K \mathrm{~s}$ and $J-K \mathrm{~s}$ colours for all objects detected in all three bands in the GRB 001011 error box. Most of the sources lie in the sequence also seen for the 2MASS+USNO sources. When the selection method described above was applied, six candidates were selected, but three of them were rejected because of their non-stellar shape (using the selection filter described in Sect. 3.4).

Second epoch observations performed on Oct. 14.002914.0521 UT at the 1.54D showed that one of the three candidates had almost disappeared (see Fig. 1). The remaining two sources are compact, non-transient and have colours that are consistent with the OA colour-colour space locus. Both objects can be seen in the upper left panel of Fig. 2 as the two closest open diamonds to the GRB 001011 colours (star) and consistent with the shaded area. The magnitudes of the two objects are $R=21.04$ and $R=21.38$, so the contamination is consistent with the expected number of compact galaxies and quasars with $21<R<22$ estimated in Sect. $3.4(1+0.3 \lesssim$ number of compact galaxies + quasars $\lesssim 3+0.7)$. The colours of both objects can be reproduced by synthetic traces of quasars 


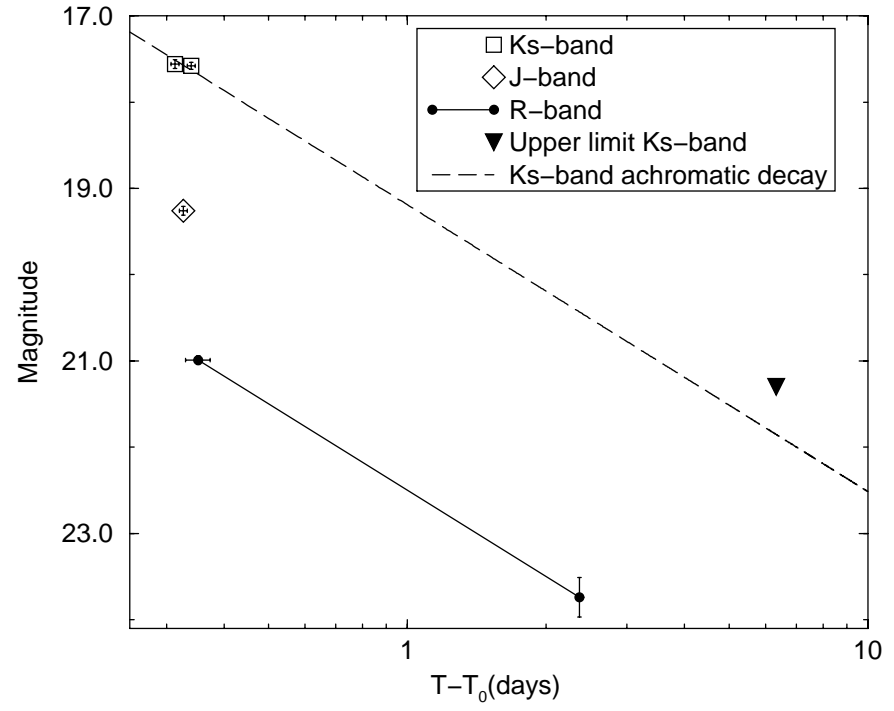

Fig. 4. The figure shows the afterglow history in the $K \mathrm{~s}, J$, and $R$-bands. The two open squares show the $K$ s-band detections and the diamond the $J$-band magnitude. The symbol size of these points has been enlarged in order to make easier the visualisation of the error bars contained in the symbols. The solid line represents the $\alpha_{R}=1.33$ decay between our two $R$-band measurements (filled circles). The triangle shows the $K$ s-band upper limit imposed on 17.9794-18.0138 UT Oct. 2000 by the NTT observations. The error bars along the horizontal axis represent the exposure time of the observations. As can be seen the second $K \mathrm{~s}$-band observation, the first $R$-band detection and the $J$-band measurement overlap in time, so they have been the basis of the colour-colour plot presented in Fig. 2 (see the observations indicated with a dagger in Table 1). The dashed line shows that the NTT upper limit is consistent with an achromatic decay $\left(\alpha_{R}=\alpha_{K \mathrm{~s}}\right)$.

or galaxies (see Fig. 2 lower left panel), so they are likely quasars or compact galaxies.

An astrometric solution based on 50 USNO A2-0 reference stars yields $\alpha_{2000}=18^{\mathrm{h}} 23^{\mathrm{m}} 04.56^{\mathrm{s}}, \delta_{2000}=$ $-50^{\circ} 54^{\prime} 15^{\prime \prime} .8$ (uncertainty $1^{\prime \prime}$. 0 ) for the OA, indicated in Fig. 1. These coordinates correspond to the Galactic coordinates $l=343.686^{\circ}, b=-16.535^{\circ}$ and a Galactic colour excess $E(B-V)=0.10$ (Schlegel et al. 1998).

The position of the counterpart is fully consistent with the position of the refined $2^{\prime} \mathrm{WFC}$ radius error circle (Gandolfi et al. 2000b) and is located only 19'.5 from its centre. The counterpart turned out to be the object with the reddest $J-K$ s colour among the 90 point-like sources located within the GRB error box and detected in the three bands. The dereddened colours of GRB 001011 are $(J-K \mathrm{~s})_{\mathrm{GRB} 001011}=1.62 \pm 0.06,(R-K \mathrm{~s})_{\mathrm{GRB} 001011}=$ $3.17 \pm 0.07$, fully consistent with the dereddened colours of GRBs detected to date in the $R, J$, and $K$ s-bands and close to the trace of OA power-law SEDs (see solid straight line of Fig. 2 right upper panel). The counterpart was point-like in the optical and in the near-IR, showing the same point-spread function as neighbouring stars. In order to improve the photometry given by the automatic pipe-line more accurate photometry was carried out by means of the DAOPHOT-II photometry package. The final photometry of the counterpart is shown in Table 1 and displayed in Fig. 4. The decay indexes in the $R$ and $K$ s bands are $\alpha_{R}=1.33 \pm 0.11$ (solid line of Fig. 4) and $\alpha_{K \mathrm{~s}}=0.24 \pm 0.73$, respectively. The large error in the determination of $\alpha_{K \mathrm{~s}}$ is due to the short time-span between the two $K$ s-band detections.

In order to measure a possible deviation from an achromatic decay, we have calculated the expected magnitude difference between the second and the first epoch $K$ sband detections if $\alpha_{R}=\alpha_{K \mathrm{~s}}$ is assumed. The prediction $(\Delta K \mathrm{~s}=0.12 \mathrm{mag})$ is only $1.5 \sigma$ different from the measured magnitude difference $(\Delta K \mathrm{~s}=0.02 \pm 0.06)$, so an achromatic decay is an acceptable approximation for our two $K$ s-band detections. A power-law fit to the two $K \mathrm{~s}$-band detections, fixing an achromatic decay index $\left(\alpha_{K \mathrm{~s}}=\alpha_{R}=1.33 \pm 0.11\right.$, see dashed line of Fig. 4), yields $K \mathrm{~s}=21.85 \pm 0.35$ on 17.9794-18.0138 UT Oct. 2000. Thus, the NTT upper limit $(K \mathrm{~s}>21.3$, represented with a triangle in Fig. 4) is consistent with an achromatic fading. We conclude that our measurements are consistent with an achromatic decay, although we can not exclude a more complex $K$ s-band lightcurve, with plateau phases (e.g. GRB 000301C, Rhoads \& Fruchter 2001; GRB 010222, Masetti et al. 2001) or re-brightenings (e.g. GRB 971214; Gorosabel et al. 1998b).

Once the $R J K$ s-band quasi-simultaneous magnitudes (see magnitudes indicated with a dagger in Table 1) have been dereddened by Galactic extinction in the direction of GRB $001011(E(B-V)=0.10$; Schlegel et al. 1998) and shifted in time (assuming an achromatic fading with $\alpha=1.33$ ) to the mean epoch of the second $K$ s-band detection (12.0028 UT), a least-squares fit provides a spectral index of $\beta=1.25 \pm 0.05$. The value of $\beta$ has been derived without considering intrinsic extinction, which is unknown for GRB 001011. Therefore, $\beta=1.25$ has to be considered as an upper limit to the actual OA spectral index.

Given that we have measured $\alpha$ and constrained the value of $\beta$, these values can be compared to the predictions given by several afterglow models. However, given the poor coverage of the light curve and the absence of an estimate of the intrinsic extinction we stress that only weak conclusions on the afterglow physical properties can be deduced from our data. If we assume an unextincted afterglow then the upper limit on $\beta$ would be close to the actual spectral index. According to the adiabatic expansion of a spherical afterglow in the slow-cooling regime (Sari et al. 1998), $\alpha=3 \beta / 2$ (for $\nu<\nu_{\mathrm{c}}$ ) and $\alpha=(3 \beta-1) / 2$ (for $\nu>\nu_{\mathrm{c}}$ ) is expected. Thus, the prediction of $\alpha$ given for $\nu<\nu_{\mathrm{c}}$ is $\alpha=1.88 \pm 0.08$, which is $4 \sigma$ away from the measured value. However, the predicted value given for $\nu>\nu_{\mathrm{c}}$ is $\alpha=1.38 \pm 0.08$ fully consistent with the measured value of $\alpha=1.33 \pm 0.11$. Besides, the derived value of the electron power-law index, $p=2 \beta=2.5 \pm 0.1$, is in agreement with those obtained for other afterglows. For the more general and common case of an extincted afterglow the value of $\beta$ would be decreased and it could be compatible with the predictions given by the spherical 


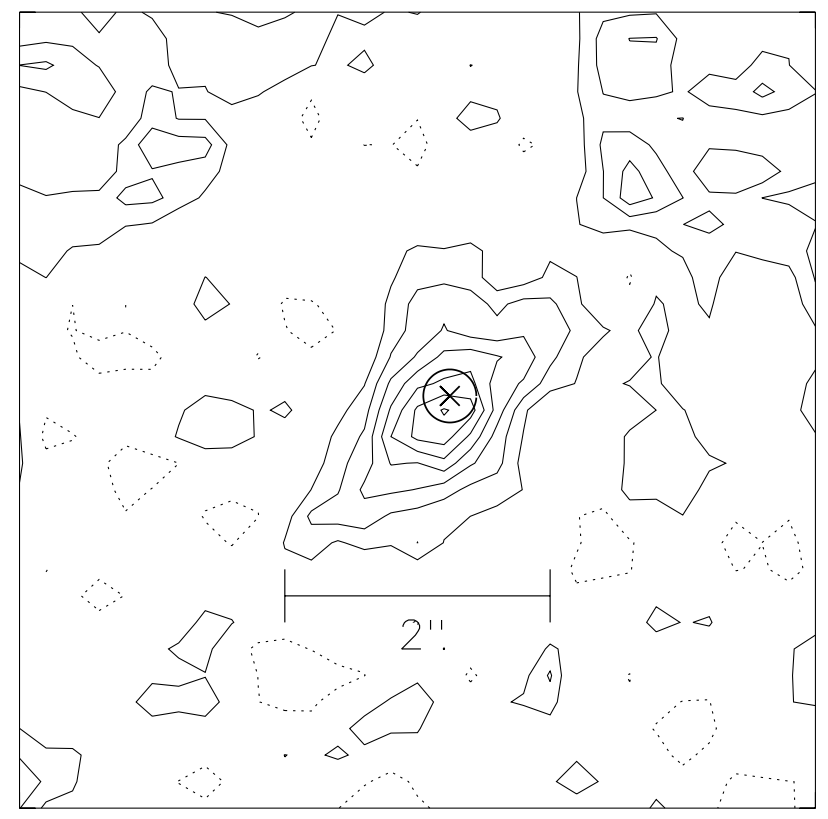

Fig. 5. The figure shows a contour plot of the co-added $R$-band images taken in May 2001 with the VLT. The total exposure time is $2400 \mathrm{~s}$ with a mean seeing of 0. . 9 . The cross shows the position of the optical counterpart, fully consistent with the object. The object is clearly elongated in the North-West direction. The image has been smoothed with a $3 \times 3$ pixel boxcar filter. North is to the top and East to the left.

adiabatic expansion for the case of $\nu<\nu_{\mathrm{c}}$. The measured value of $\beta=1.25 \pm 0.05$ is also explainable in the context of the cannonball model (Dado et al. 2001).

\subsection{The host galaxy}

When all the $R$-band images taken in April 2001 at the $1.54 \mathrm{D}$ were co-added, the presence of a very faint extended object $\sim 3 \sigma$ above the background was noticeable. Further deep optical observations carried out one month later with the VLT confirmed the presence of an object (detected at $8 \sigma$ significance) with $R=25.38 \pm 0.25$ measured in a $33^{\prime \prime} 0$ diameter circular aperture. The coordinates of the object are $\alpha_{2000}=18^{\mathrm{h}} 23^{\mathrm{m}} 04.58^{\mathrm{s}}, \delta_{2000}=-50^{\circ} 54^{\prime} 16^{\prime \prime} .0$ (uncertainty $0^{\prime \prime} .5$ ), which is $0^{\prime \prime} .28$ from the OA position. Thus, the OA seems to be centred on the extended object. This strongly suggests that the object is the host galaxy of GRB 001011. A contour plot of the object and the position of the OA can be seen in Fig. 5. The host galaxy is elongated in the North West-South East direction $\left(\mathrm{PA}=-45^{\circ}\right)$.

\section{Discussion}

The colour-colour discrimination pipe-line presented in this paper makes use of the $R, J$, and $K$ s-band magnitudes. However, it can be applied using other bands as well. The best choice of the bands would be those where most of the curved stellar spectra are as distant as possible from the afterglow power-law SEDs. A detailed study of the optimal configuration of near-IR + optical filters is however beyond the scope of the present paper. The method can also be generalised to a higher number of filters which would allow one to construct an $N$-dimensional colour-colour space.

Obviously, the colour-colour selection is only useful for GRBs that have optical and/or near-IR afterglows. However, with $8 \mathrm{~m}$ class telescopes the technique can easily be used for bursts as faint as $R=24$. Hopefully this technique will therefore facilitate the detection of optical and/or near-IR afterglows from a larger fraction of well localised GRBs than the $\approx 30 \%$ during the latest $3-4$ years (Fynbo et al. 2001b).

Another possible bias could affect the selection of high redshift afterglows. As long as the Lyman- $\alpha$ forest does not enter into the bluest observed band the method does not introduce any redshift selection bias. At a redshift $z \gtrsim 3.7$ the Lyman- $\alpha$ forest enters the $R$-band, and the spectrum is no longer well described by a single power-law SED. However, the effect would only make the OAs redder in $R-K \mathrm{~s}$ and thereby move the OAs further away from the non-transient sources.

Therefore, as long as the object is detected in the $R$ band $\sim 5 \sigma$ above the sky background, the blanketing does not represent any inconvenience to locate and distinguish objects in the colour-colour diagram. Conversely, it would help to discriminate a candidate from the nontransient sources in the GRB error box, at least up to a given upper limit in the redshift. For higher values of the redshift where the blanketing effect makes that the $R$-band detection is below $5 \sigma$, then the large photometric errors would be a difficulty to distinguish the OA from the rest of the objects. In this regime the blanketing starts to represent an inconvenience, rather than an advantage. For even larger redshift values the blanketing effect can be so severe that the afterglow is likely to remain undetected in the $R$-band.

In the case of GRB 001011 the $R$-band detection is about $2.5 \mathrm{mag}$ above the detection limit of the image, hence we could have tolerated a blanketing effect up to $z \lesssim 5.8$ (see Fig. 3). We estimate that for the set of three images (1.54D $R$-band, NTT $J$ and $K$ s-band) used in the present study, the Lyman- $\alpha$ blanketing helps the discrimination of the afterglow in the redshift range $3.7<z<5.3$. For a redshift range $5.3<z<5.8$ the GRB 001011 $R$-band detection would be below $5 \sigma$ having $R$-band photometric errors above $\sim 20 \%$, so the efficiency of the pipeline goes down. For redshifts $z \gtrsim 5.8$ the blanketing would make the GRB 001011 afterglow undetectable for our $R$-band images. For other limiting magnitudes and combinations of filters, the corresponding redshift ranges will be different.

Obviously, the former discussion is only dealing with the impact of the blanketing on the colours of GRB 001011, and it has not considered the monochromatic cosmological dimming factor given by $D_{\mathrm{L}}(z)^{-2} \times$ $(1+z)^{\alpha-\beta-1}$, where $D_{\mathrm{L}}(z)$ is the luminosity distance. This effect would enlarge the photometric errors of the high 
redshift afterglows and hence complicate their discrimination (see Lamb \& Reichart 2000 for a detailed discussion on the effect of the monochromatic cosmological dimming factor).

The application of the method to very reddened afterglows which are bright in the near-IR but with no detectable emission in the optical is possible as long as a constraining lower limit on the $R-K \mathrm{~s}$ colour can be derived. This could happen under conditions of high extinction or with extremely high redshift bursts having $5.8 \lesssim z \lesssim 8$. The pipe-line considers the potential case of highly extincted and/or redshifted afterglows creating a list of sources only detected in the near-IR (both $J$ and $K$ s bands), and hence absent in the optical image.

The efficiency of the pipe-line in the $5.8 \lesssim z \lesssim 8$ redshift range depends on the constraint imposed by the $R$-band image. As can be seen in Fig. 2 for an $R$-band limiting magnitude of $R>K \mathrm{~s}+2$ a combined detection in both the $J$ and $K \mathrm{~s}$ bands would be able to distinguish the shaded area from the rest of the colour-colour space. The discrimination of undetected optical sources in the $R<K \mathrm{~s}+2$ region is uncertain. For $z \gtrsim 8$ the Lyman- $\alpha$ blanketing also affects the $J$-band and a single analysis becomes much more complicated.

Finally, there is a bias against OAs situated in bright host galaxies, both because the flux of the underlying galaxy may move the integrated flux of OA + galaxy away from the shaded region in Fig. 2 and because the galaxy will make the OA look extended.

\section{Conclusion}

We present a colour based selection pipe-line of OA candidates that only requires three quasi-contemporaneous images. The colour selection software is designed to be used in parallel with, or as input to, the normal technique, searching for transient sources through the comparison of observations from (at least) two epochs or with the DSS. Furthermore, it is a method that allows a fast identification of candidates for follow-up spectroscopy also for OAs that are fainter than the DSS limit at the time of first optical/near-infrared observations. Thus, the technique is not meant to replace the normal procedure, but to complement it. The combination of colour-colour and variability information could be a very powerful mean of doing automated OA discovery, with great potential for forthcoming missions like Swift.

The technique has several advantages; it can be applied any time after the gamma-ray event, using a single set of images in different filters, and it does not strictly require absolute photometric calibration. Another additional advantage is that the method is independent of the redshift, at least for redshifts with a negligible Lyman- $\alpha$ blanketing along the line of sight.

In the case that the discrimination method is based on the $R, J$, and $K$ s magnitudes, it becomes independent of the redshift for $z \lesssim 3.7$. For redshifts $3.7 \lesssim z \lesssim 5.3$ the possible blanketing effect along the line of sight helps the colour discrimination. In the range $5.3 \lesssim z \lesssim 5.8$ the blanketing introduces considerable errors in the $R$-band photometry and it starts to be an inconvenient. For $5.8 \lesssim$ $z \lesssim 8$ the efficiency is still valid as long as a constraining lower limit on the $R-K$ s colour can be derived $(R>$ $K \mathrm{~s}+2)$. For extremely high redshift afterglows $(z \gtrsim 8)$ the colour-colour discrimination technique is uncertain.

This kind of analysis is very suitable for small GRB error boxes such as the ones reported by the NFI of BeppoSAX or for the ones that HETE-II is expected eventually to determine, in which case the field contamination of quasars and compact galaxies is small.

Using this method we discovered the GRB 001011 afterglow. The GRB 001011 optical afterglow evolution is consistent with a decay index of $\alpha_{R}=1.33 \pm 0.11$. With no corrections for the intrinsic absorption, we derived a spectral index of $\beta=1.25 \pm 0.05$. Therefore, this value of $\beta$ has to be considered as an upper limit to the unextincted afterglow spectral index. If we assume negligible intrinsic absorption, the values of $\alpha$ and $\beta$ are consistent with a spherical afterglow model with an electron energy index $p=2.5$. These values also would indicate that the cooling break, $\nu_{\mathrm{c}}$, was located at frequencies lower than the $R$-band $\sim 8$ hours after the gamma-ray event. This would make from GRB 001011 a very interesting system, since in most cases $\nu_{\mathrm{c}}$ is higher than the optical frequencies at early times.

Images taken 7 months after the burst reveal an elongated object with $R=25.38 \pm 0.25$ fully consistent with the OA position, likely the host galaxy of GRB 001011 .

Acknowledgements. J. Gorosabel acknowledges support from the ESO visitors program and also the receipt of a Marie Curie Research Grant from the European Commission. We acknowledge the availability of the 2MASS and USNO catalogues. This work was supported by the Danish Natural Science Research Council (SNF). We are very grateful to I. J. Danziger for helpful comments. We thank B. Montesinos and D. Barrado y Navascués for fruitful discussions on the contamination by brown dwarfs. The observations presented in this paper were obtained under the ESO Large Program 165.H-0464. We appreciate the useful and helpful comments of the referee, Dr. G. Grant Williams.

\section{References}

Andersen, M. I., Hjorth, J., Pedersen, H., et al. 2000, A\&A, 364, L54

Boella, G., Butler, R. C., Perola, G. C., et al. 1997, A\&AS, 122,299

Boyle, B. J., Jones, L. R., \& Shanks, T. 1991, MNRAS, 251, 482

Bonnell, J. T., \& Klebesadel, R. W. 1996, in Huntsville Gamma-Ray Burst Third Symposium, ed. C. Kouveliotou, M. F. Briggs, \& G. F. Fishman, AIP Con. Proc., 384, 977

Castro-Tirado, A. J., Gorosabel, J., Benítez, N., et al. 1998, Science, 279, 1011

Castro Cerón, A. J., Gorosabel, J., Castro-Tirado, A. J., et al. 2001, A\&A, in preparation

Costa, E., Frontera, E., Heise, J., et al. 1997, Nature, 387, 783 
Dado, S., Dar, A., \& De Rújula, A. 2001, A\&A, submitted [astro-ph/0107367]

Diercks, A. H., Deutsch, E. W., Castander, F. J., et al. 1998, ApJ, 503, L105

Fan, X., Knapp, G. R., Strauss, M. A., et al. 2000, AJ, 119, 928

Fontana, A., D'odorico, S., Polo, F., et al. 2000, AJ, 120, 2206

Frail, D. A., Kulkarni, S. R., Nicastro, S. R., Feroci, M., \& Taylor, G. B. 1997, Nature, 389, 261

Frail, D. A., Kulkarni, S. R., Sari, R., et al. 2001, Nature, submitted [astro-ph/0102282]

Francis, P. J., Hewett, P. C., Foltz, C. B., et al. 1991, ApJ, 373,465

Fruchter, A. S. 1999, ApJ, 512, L1

Fynbo, J. U., Gorosabel, J., Dall, T. H., et al. 2001a, A\&A, 373,796

Fynbo, J. U., Jensen, B. L., Gorosabel, J., et al. 2001b, A\&A, 369,373

Gaffey, M. J., Bell, J. F., Hamilton Brown, R., et al. 1993, Icarus, 106, 573

Galama, T. J., Vreeswijk, P. M., van Paradijs, J., et al. 1998, Nature, 395, 670

Gandolfi, G., et al. 2000a, GCN circular \# 846

Gandolfi, G., et al. 2000b, GCN circular \# 847

Garnavich, P. M., Jha, S., Pahre, M. A., et al. 2000, ApJ, 543,61

Gorosabel, J., \& Castro-Tirado, A. J. 1998, A\&A, 337, 691

Gorosabel, J., Castro-Tirado, A. J., Wolf, C., et al. 1998a, A\&A, 339, 719

Gorosabel, J., Castro-Tirado, A. J., Willott, C. J., et al. 1998b, A\&A, 335, L5

Gorosabel, J., Hjorth, J., Pedersen, H., et al. 2000, GCN circular \# 849

Hartwick, F. D. A., \& Schade, D. 1990, ARA\&A, 28, 437

Hjorth, J., Holland, S., Courbin, F., et al. 2000a, ApJ, 534, L147

Hjorth, J., Holland, S., Courbin, F., et al. 2000b, ApJ, 539, L75

Huang, J.-S., Thompson, D., Kümmel, M. W., et al. 2001, A\&A, 368, 787

Jensen, B. L., Fynbo, J. U., Gorosabel, J., et al. 2001, A\&A, 370,909

Kinney, A. L., Calzetti, D., Bohlin, R. C., et al. 1996, ApJ, 467, 38

Kirkpatrick, J. D., Reid, I. N., Liebert, J., et al. 1999, ApJ, 519,802

Lamb, D. Q., \& Reichart, D. E. 2000, ApJ, 536, L1

Landolt, A. U. 1992, AJ, 104, 340

Lattimer, J. M., \& Schramm, D. N. 1974, ApJ, 192, L145

Luginbuhl, C. B., Vrba, F. J., Hudec, R., et al. 1995, Ap\&SS, 231, 289

Madau, P. 1995, ApJ, 441, 18

Masetti, N., Bartolini, C., Bernabei, S., et al. 2000, A\&A, 359, L23

Masetti, N., Palazzi, E., Pian, E., et al. 2001, A\&A, 374, 382

McCheyne, R. S., Eaton, N., \& Meadows, A. J. 1985, Icarus, 61,443
Mészáros, P. 2001, Invited talks at the 20th Texas Symp. on Relativistic Astrophysics, Austin, Texas, Dec. 2000; and at the 2nd Rome Workshop on GRB in the Afterglow Era, Rome, to appear in Rome Conf Procs. and in AIP Conf. Proc., ed. J. C. Wheeler, \& H. Martell [astro-ph/0103204] Metzger, M. R., Djorgovski, S. G., Kulkarni, S. R., et al. 1997, Nature, 387,878

Mink, D. J. 1997, ASP Conf. Ser., 125, 249

Motch, C., Hudec, R., \& Christian, C. 1990, A\&A, 235, 185

Møller, P., \& Jakobsen, P. 1990, A\&A, 228, 299

Palazzi, E., Pian, E., Masetti, N., et al. 1998, A\&A, 336, L95

Paczyński, B. 1998, ApJ, 494, L45

Pedersen, H., Jaunsen, A. O., Grav, T., et al. 1998, ApJ, 496, 311

Persson, S. E., Murphy, D. C., Krzeminski, W., Roth, M., \& Rieke, M. J. 1998, AJ, 116, 2475

Pickles, A. J. 1998, PASP, 110, 863

Piran, T. 2001, Gamma-Ray Bursts-When Theory Meets Observations, Invited talk Texas Symposium [astro-ph/0104134]

Ramaprakash, A. N., Kulkarni, S. R., Frail, D. A., et al. 1998, Nature, 393, 43

Rhoads, J. E., \& Fruchter, A. S. 2001, ApJ, 546, 117

Rhoads, J. E. 2001, ApJ, 557, 943

Sari, R., Piran, T., \& Narayan, R. 1998, ApJ, 497, L17

Schlegel, D. J., Finkbeiner, D. P., \& Davis, M. 1998, ApJ, 500, 525

Šimon, V. R., Hudec, G., Pizzichini, \& Masetti, N. 2001, A\&A, submitted [astro-ph 0108416]

Sokolov, V. V., Kopylov, A. I., Zharykov, S. V., Kurt, V. G., \& Berezin, A. V. 1995, Ap\&SS, 231, 343

Stetson, P. 1987, PASP, 99, 191

Stetson, P. 1997, Users Manual for DAOPHOT-II

van Paradijs, J., Groot, P. J., Galama, T., et al. 1997, Nature, 386,686

van Paradijs, J., Kouveliotou, C., \& Wijers, R. A. M. J. 2000, ARA\&A, 38, 379

Véron-Cetty, M.-P., \& Véron, P. 2001, A\&A, 374, 92

Vrba, F. J., Hartmann, D. H., \& Jennings, M. C. 1995, ApJ, 446, 115

Vreeswijk, P. M., Galama, T. J., Owens, A., et al. 1999, ApJ, 523,171

Vreeswijk, P. M., Fruchter, A. S., Kaper, L., et al. 2001, ApJ, 546,672

Warren, S. J., Hewett, P. C., Irwin, M. J., \& Osmer, P. S. 1991, ApJS, 76, 1

Wolf, C., Meisenheimer, K., Röser, H.-J., et al. 1999, A\&A, 343,399

Wolf, C., Meisenheimer, K., \& Röser, H.-J. 2001a, A\&A, 365, 660

Wolf, C., Meisenheimer, K., Röser, H.-J., et al. 2001b, A\&A, 365,681

Woosley, S. E. 1993, ApJ, 405, 273

Zharykov, S. V., Kopylov, A. I., \& Sokolov, V. V. 1995, Ap\&SS, 231,347 